\title{
A MEMS Capacitor with Improved RF Power Handling Capability
}

\author{
D. Girbau ${ }^{1}$, N. Otegi ${ }^{2}$, L. Pradell ${ }^{1}$, A. Lázaro ${ }^{3}$ \\ ${ }^{1}$ Universitat Politècnica de Catalunya, Signal Theory and Communications Department, 1-3 Jordi Girona St., 08034 \\ Barcelona, Spain. Tel. +34 934016834 \\ ${ }^{2}$ University of the Basque Country, Electricity and Electronics Department, 48080 Bilbao, Spain \\ ${ }^{3}$ Universitat Rovira i Virgili, Electronics, Electrical and Automatics Engineering Department, 43007, Tarragona, Spain.
}

\begin{abstract}
This paper presents a structure of MEMS capacitor providing independence of its nominal capacity and tuning range from the applied RF signal power. The capacitor includes a third parallel plate acting as an electrode to which an extra DC voltage is applied to compensate for the self-actuation effect. This means that the device can be used in many applications working under different RF power conditions, without changing its performance - nominal capacity and tuning range Capacitor design concept and simulations are provided. It has been manufactured using a standard surfacemicromachining MEMS technology. Experimental results are presented, validating the concept and demonstrating its feasibility and advantages.
\end{abstract}

\section{INTRODUCTION}

RF MEMS variable capacitors are being considered for next generations of wireless communication systems. Potential advantages of these devices are reconfigurability, capacity of integration and no DC power consumption (in the most common electrostatic actuation case). Some other characteristics, which not long time ago seemed to be a drawback (as for example tuning range [1] and reliability [2]) have demonstrated an outstanding performance. But concerning RF power handling capability, this is one of the unsolved problems in electrostatically-actuated parallel-plates MEMS variable capacitors. While some authors have theoretically studied the effects of RF power on MEMS capacitors [3], other works have considered this effect on switches [4], proposing different solutions to improve its performance, and empirically demonstrating their viability [5].

The most important property of MEMS is offering reconfigurability to the circuits. This reconfigurability is an added value when it can be fully controlled, but in the case of RF power applications, it may translate into a drawback, as the MEMS device changes its performance as a function of RF power. This unwanted behaviour can be diminished, but not completely overcome, by increasing the total stiffness $(K)$. This solution is acceptable in capacitive switches, as the ON state capacity is in the range of $\mathrm{fF}$, but it is not in MEMS capacitors, because a change in RF power can produce an important change in the nominal capacity and in the tuning range. In addition to this, increasing $K$ means increasing the control voltage, which is not permitted in many applications. Moreover, the required $K$ for a specific power condition in a circuit may not be the same as the one required for another power condition in a different circuit. Thus, different MEMS designs would be required, with an additional cost in terms of re-designing, manufacturing and testing.

In this paper, it is proposed to use a MEMS variable capacitor [1] with three parallel-plates to compensate for the effects of power handling on the device, making its characteristics independent of the injected RF power, with three objectives:

1) To obtain a capacitor with a constant nominal capacity versus RF power.

2) To keep the device tuning range invariable with RF power.

3) To provide flexibility: the capacitor can be used in different circuits working under different power conditions, without needing to be re-designed.

\section{EFFECTS OF RF VOLTAGE ON A TWO PARALlEL- PlATES MEMS VARIABLE CAPACITOR}

Two effects limit RF power handling in MEMS capacitors: self-actuation and thermal effects due to RF current. Self-actuation, the effect considered in this work, is the attractive force that appears on a mobile membrane due to the RF rms voltage [4], causing the membrane to deflect in a similar manner to an applied DC bias. If a RF signal $V_{\text {RFrms }} \sqrt{2} \cos (\omega t)$ is simultaneously applied to the DC bias $V_{D C}$, the resulting electrostatic force can be written as:

$$
F_{e}=-\frac{1}{2} \frac{\partial C}{\partial x}\left(V_{D C}^{2}+V_{R F r m s}^{2}\right)
$$

where $C$ is the RF capacity and $x$ the displacement of the mobile membrane. The consequence of this self-actuation effect is shown in Fig.1.a and Fig.1.b, where the displacement of the mobile membrane and the capacity variation of a two parallel plates capacitor are shown as a function of $V_{D C}$ and $V_{\text {RFrms }}$ voltages, respectively. Two consequences of the RF self-actuation can be appreciated: an increase of the nominal capacity and a decrease of the tuning range.

On the other hand, RF current results in thermal heating of the membrane causing the material to expand and the membrane to deflect. This is an effect which in low-loss devices is much less significant than self- 
actuation, in spite it should be taken into account mainly under high power conditions [4], and is not considered here.

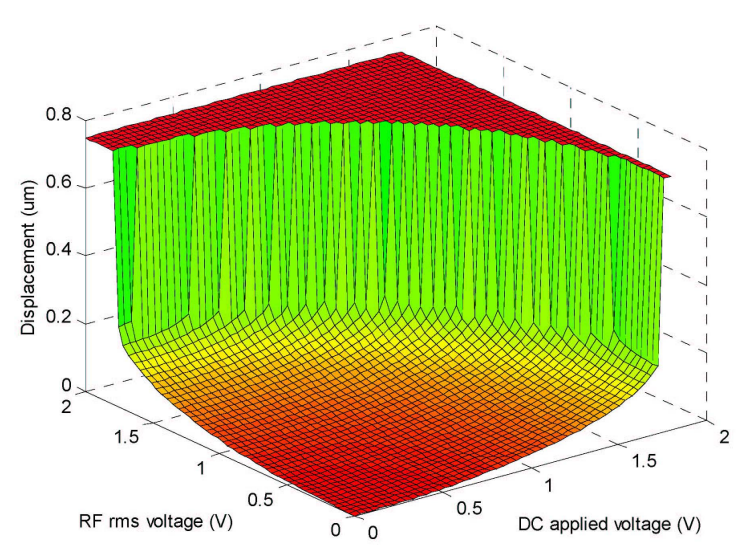

Fig.1.a. Displacement of the mobile membrane as a function of DC bias $V_{D C}$ and of RF rms voltage $V_{R F r m s}$.

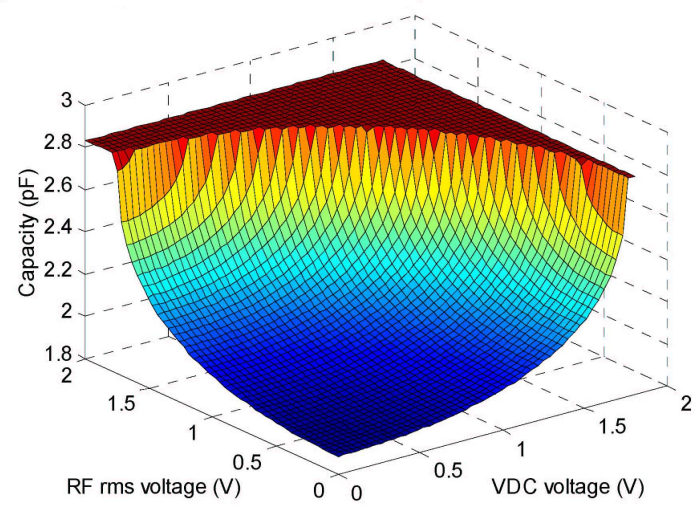

Fig.1.b. Capacity variation in a two parallel-plates MEMS capacitor as a function of DC bias $V_{D C}$ and of RF rms voltage $V_{R F r m s}$. Capacitor parameters: see section III.

\section{CAPACITOR TOPOLOGY AND PERFORMANCE ANALYSIS}

\section{A. Capacitor Concept}

The solution proposed in this paper consists of adding a third parallel plate and a second control voltage $V_{D C d w n}$, as shown in Fig.2, being the mobile plate the one in the middle. The two parallel-plates capacitor is built between the middle and top plates. A new electrostatic force appears between the mobile plate and the third added bottom plate. This means that any value of RF voltage causing self-actuation can theoretically be compensated by biasing the third plate. This second bias provides a suitable force in order to keep the mobile membrane in the right position $x_{D C}$-determined by DC biasing $V_{D C}$, and making it independent from the RF voltage. The effect of adding a restoring force can be observed in Fig.3.a. In this way, the limit in RF power handling will increase up to the limit imposed by current density, much higher than the one imposed by self-actuation [4]. The latter depends basically on the materials and shape of the structures. Another important advantage of this solution is no DC current consumption, as it is based on adding an electrostatic force-DC voltage-.

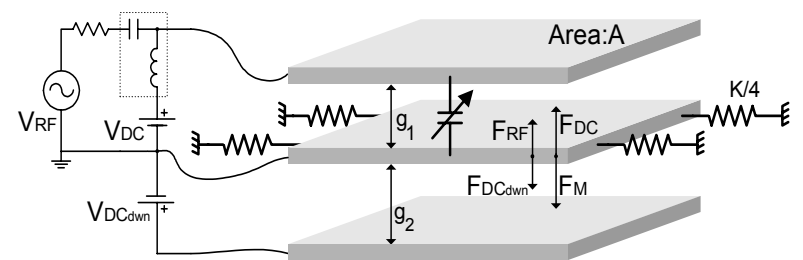

Fig.2. Schematic of the proposed MEMS capacitor to improve RF power handling.

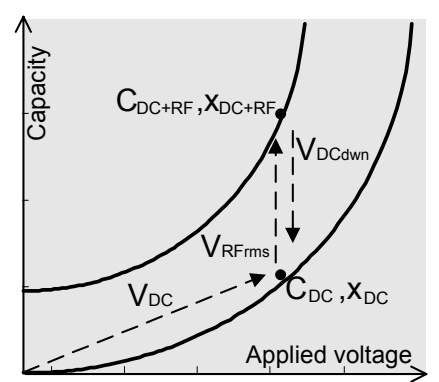

Fig.3.a. Effect of adding the restoring voltage $V_{D C d w n}$.

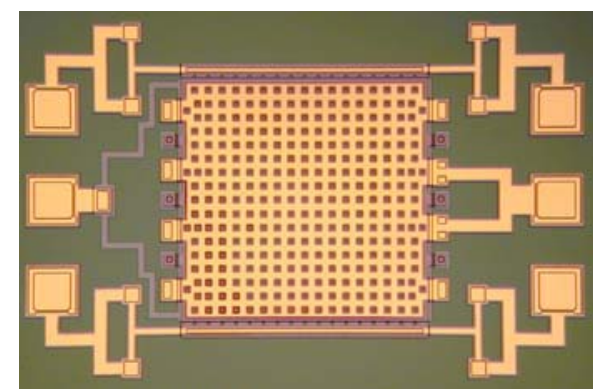

Fig.3.b. Photograph of the manufactured capacitor

The 1-D equation that rules the steady-state position of the membrane is:

$$
\begin{aligned}
& \sum F_{\text {electrostatic }}=F_{D C}+F_{R F}+F_{D C d w n}=F_{M} \\
& \frac{\varepsilon A V_{D C}^{2}}{2\left(g_{1}+x\right)^{2}}+\frac{\varepsilon A V_{R F r m s}^{2}}{2\left(g_{1}+x\right)^{2}}-\frac{\varepsilon A V_{D C d w n}^{2}}{2\left(g_{2}-x\right)^{2}}=-K x
\end{aligned}
$$

being $F_{D C}$ the force caused by the DC bias signal $V_{D C}$, $F_{R F}$ the force caused by the RF signal $V_{R F r m s}$ (selfactuation), $F_{D C d w n}$ the added compensating force caused by $V_{D C d w n}, F_{M}$ the mechanical restoring force, $A$ the area and $\varepsilon$ the gap permittivity (air). The compensating voltage $V_{D C d w n}$ that has to be applied to the third plate depends on the position $x_{D C}$ where the plate has to be replaced and on the rms value of the RF voltage signal $V_{\text {RFrms }}$ :

$$
V_{D C d w n}=\frac{\left(g_{2}-x_{D C}\right)}{\left(g_{1}+x_{D C}\right)} \cdot V_{R F r m s}
$$

\section{B. Simulated Performance}

Table I shows the parameters of the designed threeplates capacitor. It has been manufactured with the commercially available surface-micromachining process PolyMUMPS $^{\mathrm{TM}}$. 
The design process for this capacitor (Fig.3.b) is similar to that of a two parallel-plates capacitor [1], but adding an extra (bottom) plate placed at a distance $g_{2}$ (air gap 2) below the middle plate. The thickness of this gap $g_{2}$ is fixed by the technology. Then, using (3), the restoring voltages $V_{D C d w n}$ are plotted as a function of RF voltages.

\begin{tabular}{|c|c|c|c|}
\hline Area $\mathrm{A}$ & $400 \times 400 \mu \mathrm{m}^{2}$ & \multirow{2}{*}{$\begin{array}{c}\text { E (Young } \\
\text { Modulus) }\end{array}$} & $165 \mathrm{GPa}$ \\
\hline Gap1 $\mathrm{g}_{1}$ & $0.75 \mu \mathrm{m}$ & $\mathrm{K}$ & $35 \mathrm{~N} / \mathrm{m}$ \\
\hline Gap2 $\mathrm{g}_{2}$ & $2 \mu \mathrm{m}$ & $\mathrm{K}$ &
\end{tabular}

TABLE I

PARAMETERS OF THE DESIGNED CAPACITOR

Fig.4 shows the displacement of the mobile membrane working as a traditional two parallel-plates $\left(V_{D C d w n}=0\right)$, for five RF rms voltages $V_{R F r m s}$. It is observed that the nominal capacity increases and the tuning range reduces as the RF voltage increases. The objective of the proposed capacitor topology is to obtain a displacement (and, therefore, capacity) corresponding to the trace of no RF power applied, independently of the effectively applied RF power.

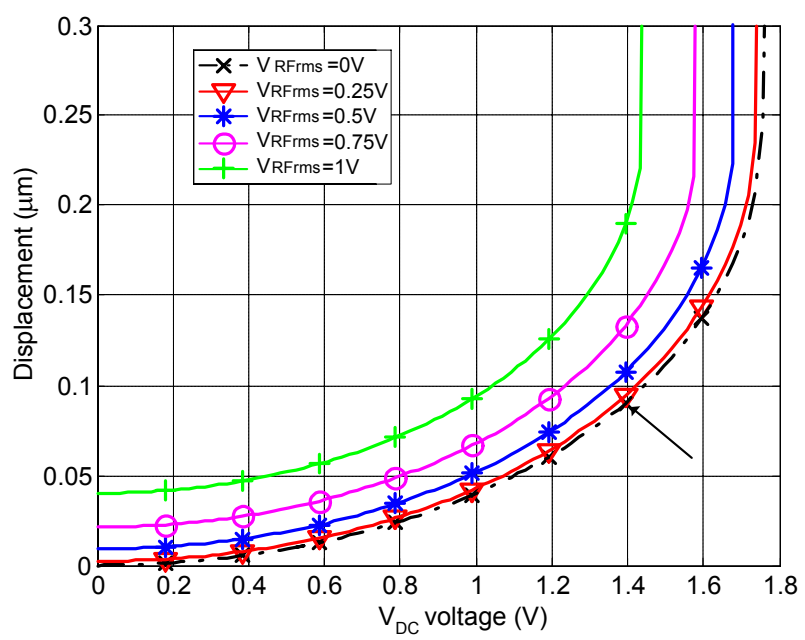

Fig.4. Simulated effect of the RF rms voltage $V_{R F r m s}$ on the mobile membrane: nominal capacity increase and tuning range decrease.

To this end, Fig.5 shows a plot of the $V_{D C d w n}$ required for the designed capacitor, as a function of the applied DC bias voltage $V_{D C}$ (which determines the membrane position $x_{D C}$ ), for the same five RF voltages as in Fig.4. As an example (see the arrows in Figs.4-6) assume an application requiring the capacitor mobile membrane in the position $x_{D C}=0.09 \mu \mathrm{m}$ (corresponding to an applied DC voltage $V_{D C}=1.4 \mathrm{~V}$ ) and a RF voltage $V_{R F r m s}=1 \mathrm{~V}$. From Fig. 4, the actual position of the membrane due to self-actuation is $x_{D C+R F}=0.19 \mu \mathrm{m}$. From Fig. 5 , for the abscise $1.4 \mathrm{~V}$ and a RF voltage of $1 \mathrm{~V}$, the required restoring voltage can be obtained: $V_{D C d w n}=3.17 \mathrm{~V}$. Applying this voltage to the third plate, provided that the capacitor is under those $\mathrm{DC}$ and $\mathrm{RF}$ conditions will restore the target position $x_{D C}$.

This effect can be seen in Fig.6, where the displacement for the condition of no RF voltage applied is plotted along with displacement for $V_{D C d w n}=3.17 \mathrm{~V}$ and $V_{\text {RFrms }}=1 \mathrm{~V}$. It can be observed that the capacity converges at a displacement of $x_{D C}=0.09 \mu \mathrm{m}$ for an applied DC voltage $V_{D C}=1.4 \mathrm{~V}$, as expected. This means that the membrane positioning and RF voltage can be made independent. In an application for which a constant power and constant position are expected (as for instance, a capacitor in a matching network) this is the right solution, because the map of the voltages to be applied to the third membrane is perfectly known (see Fig.5) as a device characteristic. In applications requiring the membrane to work at different positions (as for instance, voltage controlled oscillators), a good solution is applying a constant $V_{D C d w n}$ restoring voltage. In the presented example, a reasonable figure for $V_{D C d w n}$ would be $2.7 \mathrm{~V}$, corresponding to the restoring voltage for $V_{D C}=0 \mathrm{~V}$ in the case of $V_{R F r m s}=1 \mathrm{~V}$. Observe in Fig.6 that, although it is not an optimum solution for all membrane positions, it is much better than not applying it, in spite of a tuning range slight reduction. In this case, the nominal capacity also remains constant with RF voltage.

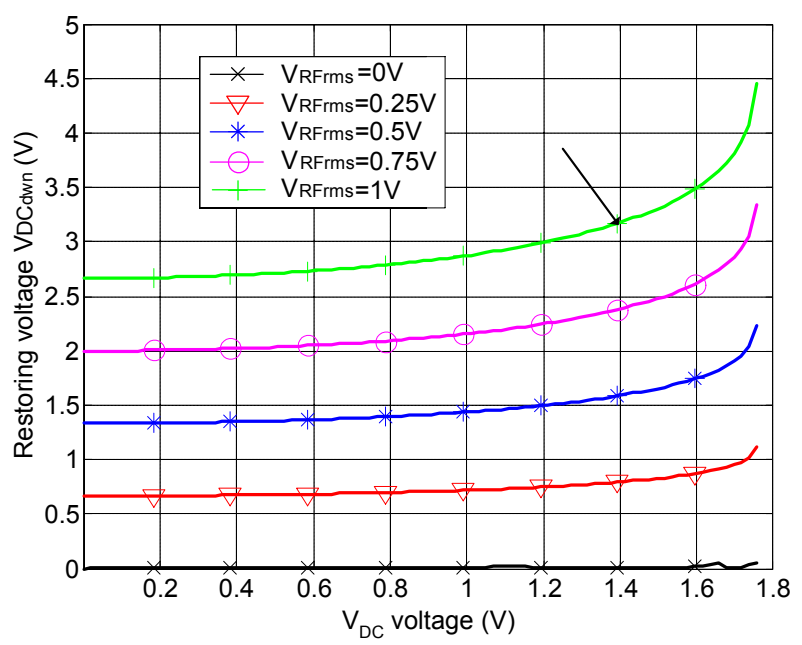

Fig.5. $V_{D C d w n}$ voltage required to restore the mobile membrane as a function of the $V_{D C}$ voltage (determined by the required $x_{D C}$ position), for five RF rms voltages $V_{R F r m s}$.

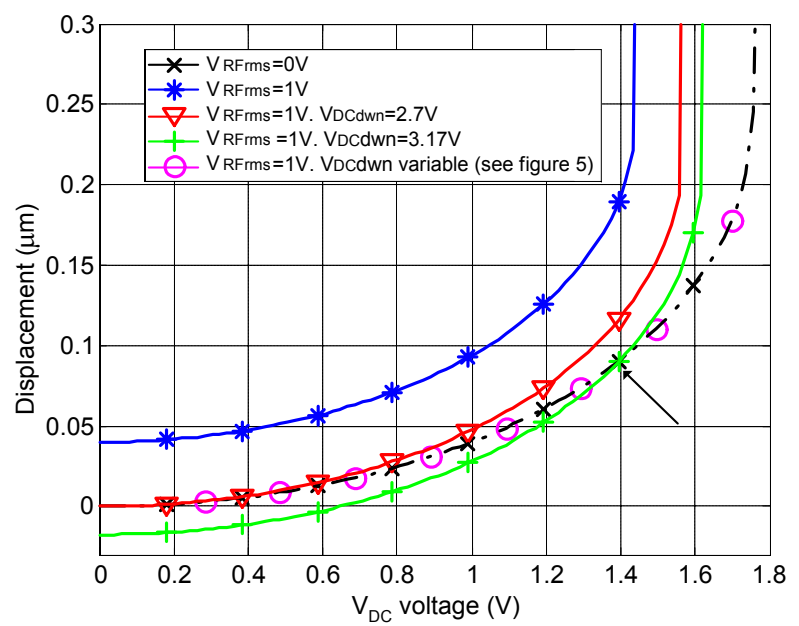

Fig.6. Simulated results of applying a variable and a constant $V_{D C d w n}$ voltage in order to restore the mobile plate to its $x_{D C}$ position. 


\section{EXPERIMENTAL RESULTS}

Measurements of RF power handling have been done at $1.5 \mathrm{GHz}$ using the system proposed in [6]. It is based on I/Q demodulation of the reflected signal in RF MEMS capacitors in order to characterize dynamics and power handling. It is advantageous in terms of allowing $\mathrm{S}$ parameters time measurement as well as suitable for high RF power signals. Fig.7 shows the measured capacity versus applied voltage in five cases. First, the capacity is measured using a HP8510 VNA with low RF power (0 $\mathrm{dBm}$ ), and the result is compared to the capacities obtained using the measurement system of [6] for RF powers of 14 and $16 \mathrm{dBm}$. A shift in the nominal capacity of $0.13 \mathrm{pF}$ in the first case and $0.25 \mathrm{pF}$ in the second can be appreciated. Finally, two more plots are shown, corresponding to the capacity excursions with an applied RF power of 14 and $16 \mathrm{dBm}$, but adding their respective $V_{D C d w n}$ restoring voltages: 3.2 and $3.6 \mathrm{~V}$. It can be seen that the nominal capacity has been restored to its correct position and the tunability made independent of the RF power. Furthermore, as it is observed in Fig.5, applying a constant voltage for a given RF power is a good option because the restoring $V_{D C d w n}$ voltage is nearly constant during all the excursion except when the capacitor is close to the pull-in limit, where the required restoring voltage has a slight increase.

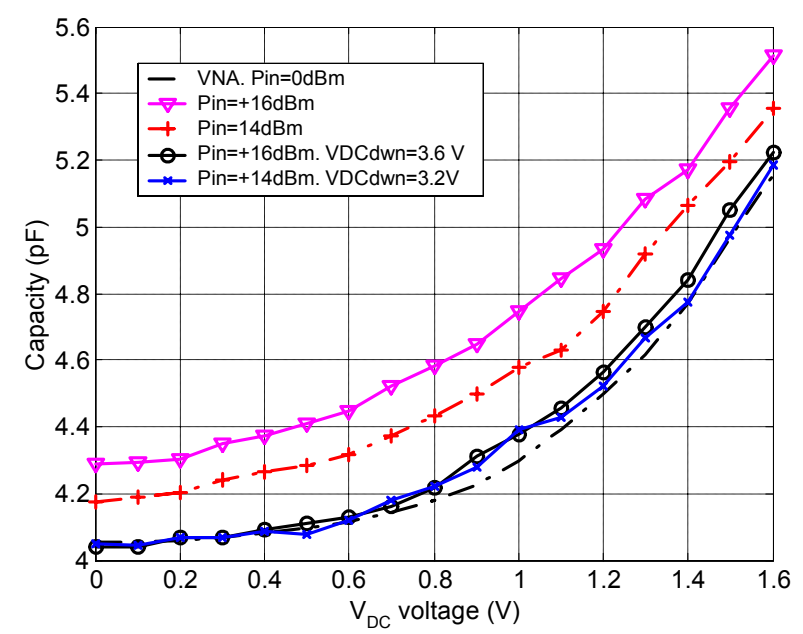

Fig. 7. Measured results. Comparison between the capacity variation at +14 and $+16 \mathrm{dBm}$ RF power with and without restoring $V_{D C d w n}$ voltages, measured with the system proposed in [6]. Comparison to the measurement with low RF power using a VNA.

The plots were obtained as an average of 2730 measurements at each point. Measurements have been done up to $1.6 \mathrm{~V}$ (below pull-in) because the capacitor remained in stiction after pull-in collapse, as there is no dielectric layer to avoid contact between electrodes.

\section{CONCLUSION}

This work has presented simulated and experimental results of a MEMS variable capacitor whose topology, based on the addition of a third parallel plate, provides a solution to the issue of RF power handling, important limitation in MEMS capacitors. With the proposed structure, the nominal capacity and the tuning range have demonstrated independence from the applied RF signal power. Design equations are provided, the capacitor performances are simulated and a practical realization is designed and tested. Experimental results show that, by applying a restoring voltage to the third plate, the nominal capacity and tuning range are made independent of RF voltage, counteracting the RF self-actuation effect.

\section{ACKNOWLEDGEMENT}

This work was supported by the Spanish Government under Grant ESP2004-07067-C03-03 (MCYT).

\section{REFERENCES}

[1] D.Girbau, A.Lázaro, L.Pradell, "Extended tuning range RF MEMS variable capacitors using electrostatic and electrothermal actuators". Proceedings of SPIE Micromachining and Microfabrication 2004, vol. 5344, pp.59-70. January, 2004.

[2] I.De Wolf, "The reliability of RF-MEMS: failure modes, test procedures and instrumentation", Proceedings of SPIE Micromachining and Microfabrication 2004, vol. 5343, pp.1-8. January, 2004.

[3] A.Cruau, P.Nicole, G.Lissorgues, C.-M.Tassetti, "Influence of RF signal power on tunable MEMS capacitors". $33^{\text {rd }}$ European Microwave Conference, Vol. 2, pp. 663-666, October 2003.

[4] J.R.Reid, L.A.Starman, R.T.Webster, "RF actuation of Capacitive MEMS Switches", 2003 MTT-S Digest, Vol.3, pp. 1919-1922, June 2003.

[5] D.Peroulis, S.P.Pacheco, L.P.B.Katehi, "RF MEMS Switches With Enhanced Power Handling Capabilities", IEEE Trans. on Microwave Theory and Techniques, Vol. 52, $\mathrm{N}^{\mathrm{o}}$ 1, pp. 59-68 January 2004.

[6] D.Girbau, A.Lázaro, L.Pradell, "Characterization of dynamics and power handling of RF MEMS using vector measurement techniques", IEEE Trans. on Microwave Theory and Techniques, Vol. 52, $\mathrm{N}^{\mathrm{o}} 11$, pp. 2627-2633 November 2004. 